\title{
Modeling and experimental methods to predict oxygen distribution in bone defects following cell transplantation
}

\author{
Christopher M. Heylman • Sharon Santoso • \\ Melissa D. Krebs • Gerald M. Saidel • \\ Eben Alsberg • George F. Muschler
}

\begin{abstract}
We have developed a mathematical model that allows simulation of oxygen distribution in a bone defect as a tool to explore the likely effects of local changes in cell concentration, defect size or geometry, local oxygen delivery with oxygen-generating biomaterials (OGBs), and changes in the rate of oxygen consumption by cells within a defect. Experimental data for the oxygen release rate from an OGB and the oxygen consumption rate of a transplanted cell population are incorporated into the model. With these data, model simulations allow prediction of spatiotemporal oxygen concentration within a given defect and the sensitivity of oxygen tension to changes in critical variables. This information may help to minimize the number of experiments in animal models that determine the optimal combinations of cells, scaffolds, and OGBs in the design of current and future bone regeneration strategies. Bone marrow-derived nucleated cell data suggest that oxygen consumption is dependent on oxygen concentration. OGB oxygen release is shown to be a time-dependent function that must be measured for accurate simulation. Simulations quantify the dependency of oxygen gradients in an avascular defect on cell concentration, cell oxygen consumption rate, OGB oxygen generation rate, and OGB geometry.
\end{abstract}

C. M. Heylman $(\bowtie) \cdot$ G. F. Muschler

Department of Biomedical Engineering, Cleveland Clinic Lerner Research Institute, 9500 Euclid Ave ND20, Cleveland, OH 44195, USA

e-mail: cheylman@uci.edu

C. M. Heylman $\cdot$ S. Santoso $\cdot$ M. D. Krebs $\cdot \leftarrow$

G. M. Saidel · E. Alsberg

Department of Biomedical Engineering, Case Western Reserve University, 319 Wickenden Building, 10900 Euclid Ave,

Cleveland, $\mathrm{OH} 44106$, USA
Keywords Oxygen consumption $\cdot$ Bone defect $\bullet \leftarrow$ Oxygen tension - Tissue engineering $\cdot$ Mathematical modeling

\section{Introduction}

Effective tissue regeneration in a bone defect is clinically essential in many settings of high-energy trauma in order to return patients to full mobility and productive life. Bone formation requires the contribution of osteogenic (bone forming) connective tissue progenitors (CTP-Os). CTP-Os represent a heterogeneous population of cells resident in bone marrow, periosteum, and other tissues that are capable of proliferating and differentiating into one or more connective tissues [13, 14]. In many clinical settings, such as fracture nonunion and bone defects, the local population of CTP-Os is suboptimal [3]. In these settings, transplantation of bone marrow-derived osteoblastic stem and progenitor cells is a promising therapeutic strategy. However, oxygen tension within a site where CTP-Os are transplanted is a critical determinant of cell survival and cell fate.

CTP-Os can be readily harvested from bone and marrow tissues. In clinical practice, they are transplanted in the form of cancellous autograft bone or bone marrow aspirates and are often combined with biocompatible scaffolds $[2,16]$. However, the environments in which these grafts are placed are often characterized by profound hypoxia, which limits the likely survival of transplanted cells. As a result, optimization of the effectiveness of such cell transplantation in the setting of bone defects and other tissue engineering constructs must be informed by a keen awareness and understanding of the biological environment into which CTP-Os and other cells are placed, particularly with respect to hypoxia $[13,23]$. 
In theory, the oxygen tension environment within a bone defect can be modulated by many interacting factors, including the vascularity of surrounding tissues, the local concentration of cells in the defect, the rate of oxygen consumption by cells within a defect, defect size or geometry, and the diffusion or convection path that is available for fluid and therefore dissolved oxygen transport through the defect. Several studies have suggested that oxygen tension and the outcome of tissue formation can also be influenced by local oxygen delivery within the defect, using oxygen-generating biomaterials (OGBs) $[1,6$, $17,18,24]$.

The goal of this project was to develop a mathematical model to simulate oxygen distribution in an established in vivo bone defect $[15,22]$ as a tool to inform and accelerate the optimization of cell therapy strategies and possible use of OGBs. This model is used to explore the relative effects of variation in defect size and OGB geometry, the number of cells, the oxygen consumption rate of transplanted cells, and the oxygen release rate from an OGB. This information is essential to minimize the number of experiments in an animal model that determine the optimal combinations of scaffold, OGB, and cell concentration, thus shortening the amount of time from bench to bedside in developing new therapies.

Previous models have defined oxygen consumption [7$10,25,28]$ and oxygen transport $[9,12,14,28]$ in tissues, but these models are not designed to inform the sensitivity to variables that may be manipulated in an established preclinical animal model to enhance clinical success. In the following sections, we first describe the design and development of the mathematical model, followed by experimental studies that were performed to provide objective data on the oxygen consumption rates of the progeny of human bone marrow-derived CTP-Os and oxygen generation rates for gas-foamed cylinders of OGB. These experimental data provide a clinically relevant set of parameters that enable examination of the effect of cell number and other parameters, which are presented in subsequent sections.

\section{Methods}

\subsection{Model development}

Our general approach was to simulate the dynamic oxygen concentration distribution in an avascular cylindrical bone defect $[15,22]$. The major components that determine the oxygen concentration in such a defect include the surrounding vascularized tissues, the cell-seeded scaffold implanted in the defect, and the OGBs implanted in the center of the cell-seeded scaffold. The key model parameters relate to the defect size, scaffold size, transplanted cell density, transplanted cell oxygen consumption rate, and OGB oxygen release rate. Though we apply this model to a cylindrical avascular bone defect, this model can be modified to apply to any defect size, transplanted cell population, OGB, and tissue site.

A mathematical model was developed that describes mass transport of oxygen in a cell-seeded scaffold occupying an avascular bone defect. In the scaffold, it is assumed that the number of cells remains constant and that the cells are distributed uniformly. Consequently, the oxygen consumption rate is also spatially uniform. Following previous experiments [15, 20, 22, 26, 27], the annular space of the scaffold (Fig. 1) is axially symmetric. The dominant transport process in the avascular annular space is diffusion of oxygen in the radial direction. In the animal model simulated [15, 22], negligible diffusion occurs axially either through the cortical bone at the bottom of the defect or through the fixation plate that covers the top of the defect. Convection is deemed negligible in the absence of a vasculature and with only minimal and irregular convection possible due to the mechanical environment of the defect. Thus, the molar concentration of oxygen changes according to:

$\frac{\partial C}{\partial t}=D \frac{1}{r} \frac{\partial}{\partial r}\left(r \frac{\partial C}{\partial r}\right)(-N R(C) \leftarrow$

where $C$ is the oxygen concentration $\left(\mu \mathrm{mol} \mathrm{ml}^{-1}\right), \mathrm{r}$ is the radial distance from the center of the cylinder $(\mathrm{cm}), D$ is a constant diffusion coefficient $\left(\mathrm{cm}^{2} \mathrm{~h}^{-1}\right), \mathrm{R}(\mathrm{C})$ is the oxygen consumption rate per cell $\left[\mu \mathrm{mol} \mathrm{h}{ }^{-1}\right.$ $\left(10^{6} \text { cells }\right)^{-1}$ ], and $N$ is the number of cells per unit volume in the scaffold (cells $\mathrm{ml}^{-1}$ ). The initial condition assumes that the oxygen in the scaffold surrounded by the OGB is in equilibrium with the environment:

$t=0: C=C_{a i r}$

This is the oxygen concentration of room air corresponding to that in an open wound at the time of surgery. At the boundary of the scaffold and OGBs, the rate of oxygen generation equals the rate of diffusion:

$r=r_{O G B}: S D \frac{\partial C}{\partial r}=V G(t)$

Here, $S$ is the surface area at $r_{\mathrm{OGB}}\left(\mathrm{cm}^{2}\right)$ and $V$ is the volume occupied by the OGBs $(\mathrm{ml})$ so that $V / S=r_{\mathrm{OGB}} / 2$. Oxygen generation is assumed to be occurring homogenously over the entire lateral surface area $(S)$ of the OGB. At the boundary of the scaffold and the vascularized bone tissue, the oxygen is in local equilibrium, so that its concentration is as follows:

$r=r_{\text {bone }}: C=C_{\text {bone }}$

The volume of the vascularized bone surrounding the avascular defect is sufficiently large relative to the volume 
Fig. 1 Model geometry: a from left to right: Photograph of canine femoral defect creation using a custom drill guide, defect creation, and CTP-loaded scaffold containing oxygengenerating biomaterials (OGBs). b Geometry of defect. Dark: Scaffold with CTPs. White: OGB with no cells. c Radial cross section of defect in bone

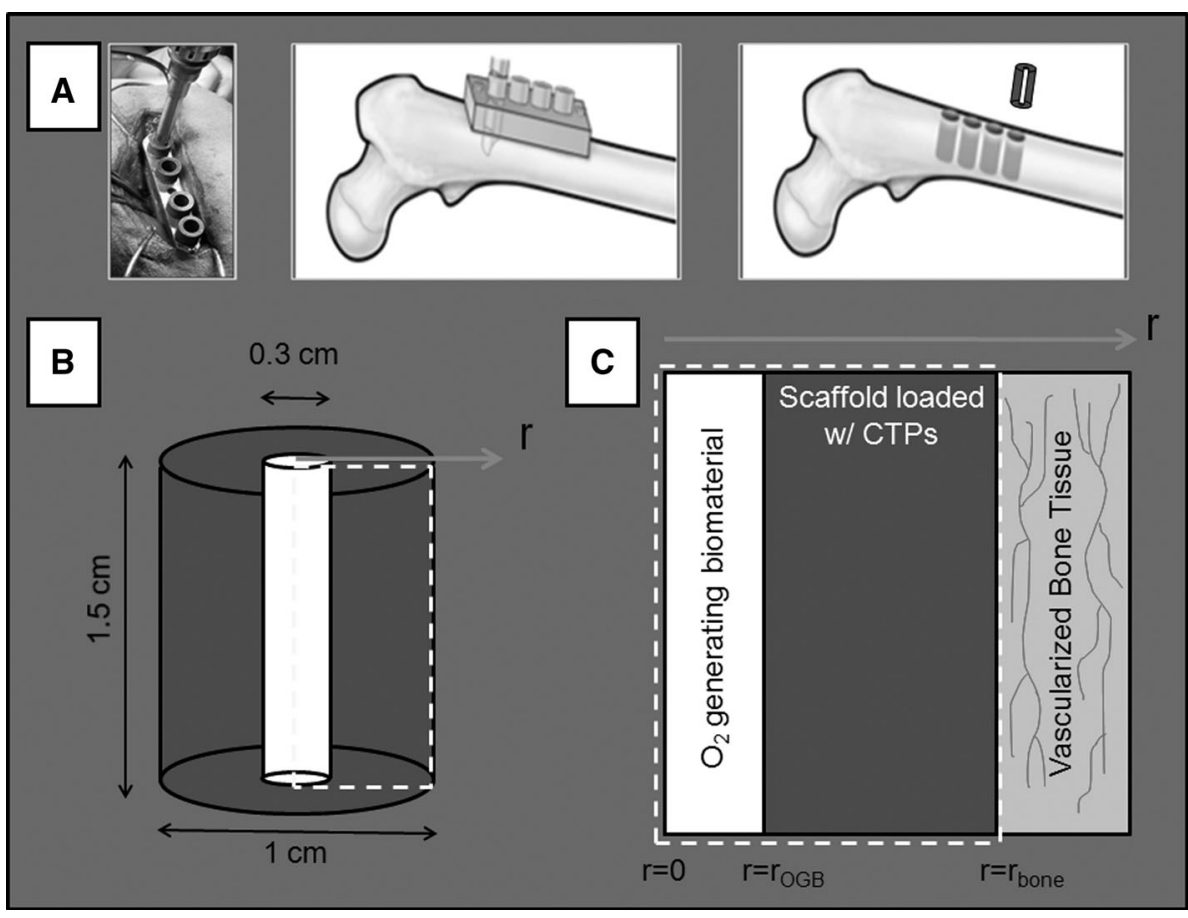

of the avascular defect such that the concentration of oxygen in the vascularized bone is assumed constant.

\subsection{Oxygen generation from oxygen-generating biomaterial (OGB)}

Gas foaming was used to fabricate cylindrical OGBs composed of poly(lactic-co-glycolic acid) (PLGA) (DURECT, Birmingham, AL) and sodium percarbonate (SPC) (Sigma Aldrich, St. Louis, MO) using published methods $[5,21]$. The PLGA used has a lactide-to-glycolide ratio of 50:50. PLGA and SPC were ground to a size range of $106-250 \mu \mathrm{m}$ and $1-63 \mu \mathrm{m}$, respectively, mixed at a mass ratio of 70:30, placed in a $3-\mathrm{mm}$ die, pressed with 1.5 tons of pressure, and transferred to a pressure cylinder. The composite was infused with $\mathrm{CO}_{2}$ at 800 psi and held for $20 \mathrm{~h}$. Pressure was then rapidly released over 1 min causing a thermodynamic instability that fuses the PLGA particles together, encapsulating the SPC particles within the bulk PLGA cylinder and resulting in an OGB cylinder $3 \mathrm{~mm}$ in diameter and $15 \mathrm{~mm}$ tall.

In vitro oxygen release from the OGB was measured using five oxygen microelectrodes (Strathkelvin Model \#1302) fitted into a RC650 respirometer (Strathkelvin Instruments, Ltd., North Lanarkshire, Scotland). OGB cylinders were placed in three respirometry wells, and control cylinders fabricated with PLGA only were placed in two separate wells. All wells were filled with sterile water. Wells were sealed and mixed with a microstir bar to prevent bubble formation from the oxygen-generating reaction. All five wells were housed within the same temperature-controlled water bath set at $37 \mathrm{C}$ to minimize well-to-well variation in temperature fluctuation. Oxygen concentration was measured continuously for 4 days. Variation in data collection from electrode to electrode was controlled by performing simultaneous calibration on all electrodes prior to each experiment to ensure accurate measurement. Calibration was checked at the conclusion of data collection to ensure minimal drift of electrode signal throughout the experiment.

The respirometry wells were opened every $12 \mathrm{~h}$ and allowed to equilibrate with room air $\left(C_{\mathrm{o}}=0.341 \mu \mathrm{mol}\right.$ $\mathrm{ml}^{-1}$ ) for the first $48 \mathrm{~h}$ to prevent oxygen from accumulating to a concentration above the dynamic range of the electrodes. The left panel of Fig. 2 shows the average oxygen concentration $\left\langle\boldsymbol{C}_{k}\left(t_{i}\right)\right\rangle\left(\mu \mathrm{mol} \mathrm{ml}{ }^{-1}\right)$ for each 12-h interval $\left(t_{\mathrm{i}}\right)$ within each well. Data are presented for each of three water samples containing OGB with SPC $(k=1)$ and each of two water samples containing PLGA only $(k=2)$. From the average oxygen concentration $\left\langle G_{\mathrm{k}}\left(t_{i}\right)\right\rangle_{\leftarrow}$ $\left(\mu \mathrm{mol} \mathrm{ml} l^{-1}\right)$, the average oxygen accumulation, $G_{k}$, per unit volume $\left(\mu \mathrm{mol} \mathrm{ml} \mathrm{m}^{-1} \mathrm{~h}^{-1}\right.$ ) of $\mathrm{k}$ for 12 -h intervals $\left(t_{\mathrm{i}+1}-t_{\mathrm{i}}, i=0,1,2, \ldots 8\right)$ is:

$G_{\mathrm{k}}=\left\{\left[\frac{V_{\text {water }}}{V_{\mathrm{k}}}\right]\right\} \frac{\left.\epsilon_{\mathrm{k}}\left(t_{i+1}\right)\right\rangle-C_{o}}{t_{i+1}-t_{i}},\left\{\begin{array}{l}\mathcal{C}_{o}=0.341, \quad 0 \leq t \leq 48 \\ C_{o}=\left\langle G_{\mathrm{k}}\left(t_{i}\right)\right\rangle, \quad 48 \leq t \leq 96\end{array}\right.$

where $V_{\text {water }}$ is the volume of water in the well $(\mathrm{ml})$ and $V_{\mathrm{k}}$ is the volume of $k(\mathrm{ml})$. The true oxygen generation rate per unit volume of OGBs, $\mathrm{G}\left(\mu \mathrm{mol} \mathrm{ml} \mathrm{l}^{-1} \mathrm{~h}^{-1}\right)$, is the 

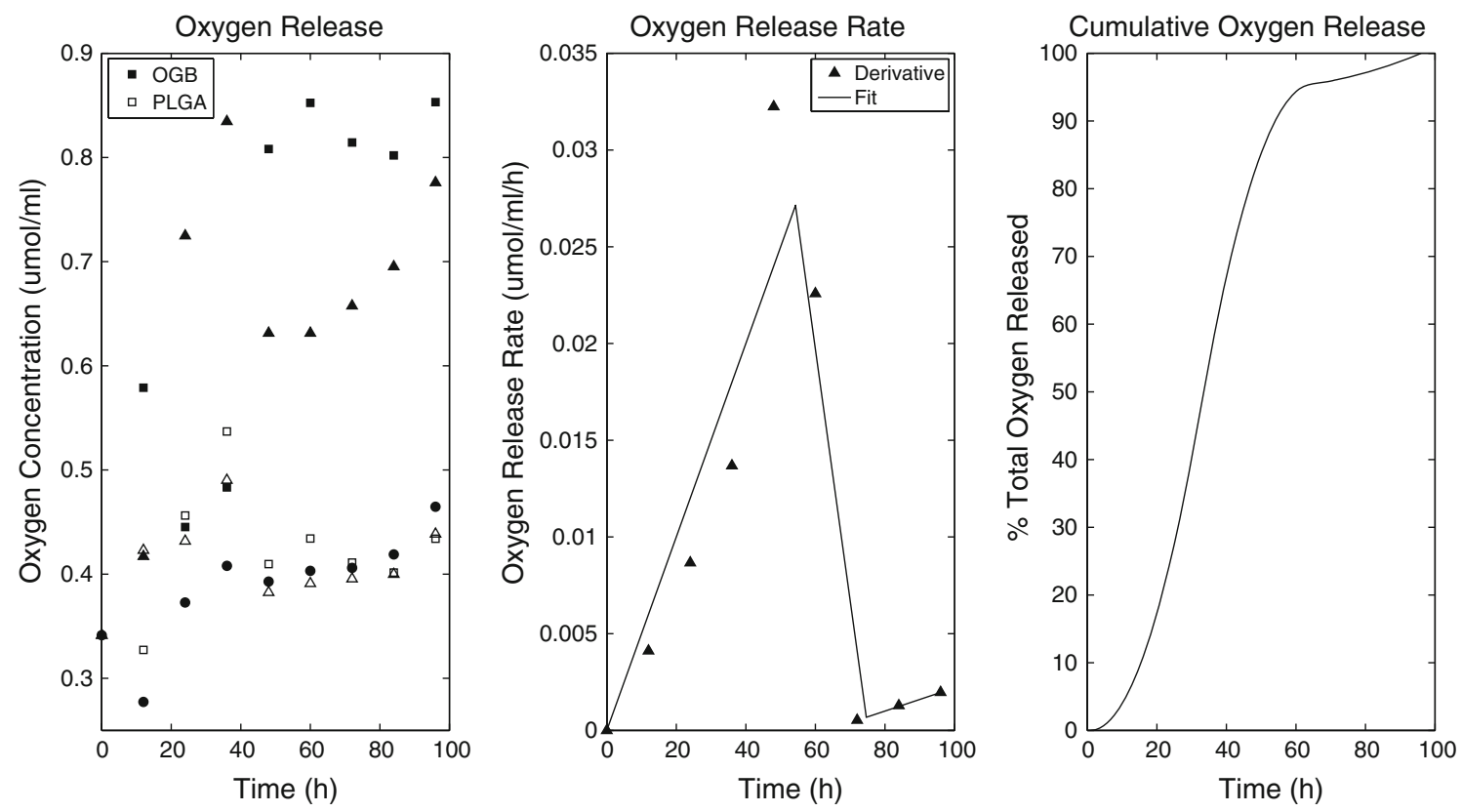

Fig. 2 Oxygen release from oxygen-generating biomaterials (OGBs). Left: Oxygen concentration in respirometer from OGB (solid symbols) or PLGA only (open symbols). Different symbol shapes represent data from each individual well. Center: Oxygen release rate as a function

difference between the rates of accumulation from OGB $\left(G_{1}\right)$ and PLGA $\left(G_{2}\right)$ :

$$
G(t)=G_{1}-G_{2}
$$

The center panel of Fig. 2 shows this average rate of oxygen generation per unit volume of the OGBs for the eight intervals and the representation of these data as a continuous, piecewise linear function:

$$
\begin{aligned}
& G(t)=\alpha t+\beta
\end{aligned}
$$

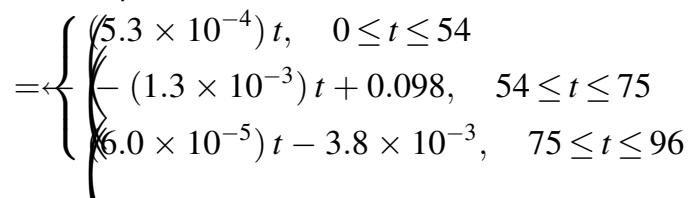

Piecewife linear segments were chosen as the simplest and best model fit to the three distinct phases of oxygen generation. The first segment, constrained to zero to prevent negative generation values for simulation, had a coefficient of determination of $R^{2}=0.847$. Each subsequent linear segment was constrained to the intersection point of the previous segment to ensure a continuous function as required for simulation. The coefficients of determination for these two segments were $R^{2}=0.952$ and 0.999 , respectively. The parameters fit to this model along with the $95 \%$ confidence interval for each parameter are as follows:

$0 \leq t \leq 54: \alpha=5.32 \times 10^{-4}\left[3.24 \times 10^{-4}, 7.40 \times 10^{-4}\right] \leftarrow$ of time (triangles: calculated derivative of oxygen concentration versus time data, line: piecewise fit of derivative data to three linear segments). Right: cumulative oxygen release as a percentage of the total oxygen released over the duration of the experiment versus time

$$
\begin{aligned}
& 54 \leq t \leq 75: \\
& \alpha=-1.32 \times 10^{-3}\left[-5.11 \times 10^{-3}, 2.47 \times 10^{-3}\right] \leftarrow \\
& \beta=9.77 \times 10^{-2}\left[-1.33 \times 10^{-1}, 3.28 \times 10^{-1}\right] \leftarrow \\
& 75 \leq t \leq 96: \alpha=5.99 \times 10^{-5}\left[3.73 \times 10^{-5}, 8.26 \times 10^{-5}\right] \leftarrow \\
& \beta= \\
& \left.\times 10^{-3}\right] \leftarrow
\end{aligned}
$$

Integration of $G(t)$ over time yields the cumulative oxygen generation (right panel). This emphasizes that more than $90 \%$ of the oxygen generated during the experiment occurred in the first $48 \mathrm{~h}$.

\subsection{Tissue harvest, cell isolation, and cell culture}

Discarded cancellous bone that was removed from the proximal femur from a human patient (male, age 64) undergoing an elective hip arthroplasty procedure was used as the cell source in a protocol approved by the Cleveland Clinic Institutional Review Board. Cancellous bone was minced with an osteotome into pieces no larger than $1 \mathrm{~mm}$ in any dimension and transferred to a 50-ml tube containing $10 \mathrm{ml}$ of $\alpha$-MEM (Sigma Aldrich, St. Louis, MO). The sample was inverted by hand 50 times, allowed to settle, and then inverted an additional 50 times to remove all suspended and loosely adherent cells from the pieces of bone. The supernatant was collected and passed through a $70-\mu \mathrm{m}$ nylon cell strainer (BD Biosciences, San Jose, CA) to remove bone 
chips and other debris. The resultant cell fraction was centrifuged at $400 \times g$ for $10 \mathrm{~min}$, and the supernatant was removed. The pellet was resuspended in $20 \mathrm{ml}$ of osteogenic media comprising $\alpha$-MEM (Sigma Aldrich, St. Louis, MO), $10 \%$ fetal bovine serum (Cambrex, Walkersville, MD), sodium ascorbate $\left(50 \mu \mathrm{g} \mathrm{ml}^{-1}\right)$ (Sigma Aldrich, St. Louis, MO), $1 \%$ antibiotic/antimycotic (Gibco, Grand Island, NY), and $10^{-8} \mathrm{M}$ dexamethasone (Sigma Aldrich, St. Louis, MO). Twelve-millimeter glass coverslips were sterilized by soaking in $70 \% \mathrm{EtOH}$ for $30 \mathrm{~min}$ followed by $30 \mathrm{~min}$ of exposure to UV radiation. The cell suspension was diluted accordingly and plated immediately on the coverslips at a density of $4.42 \times 10^{5}$ cells cm $\mathrm{cm}^{-2}$. Medium was changed twice per week, and cells were observed on a bright-field microscope for confluence. Coverslips were used for respirometry when a qualified microscopist confirmed that cells had achieved $70 \%$ confluence.

\subsection{Cellular oxygen consumption}

Closed-cell respirometry was used to measure both the consumption of oxygen by bone marrow-derived mononuclear cells and the release of oxygen from OGBs. Closed-cell respirometry was performed using a Strathkelvin 6 Electrode Respirometer fitted with six Strathkelvin 1302 Oxygen Electrodes with polypropylene membranes and controlled using a Strathkelvin Model 929 6-Channel Oxygen Meter (Warner Instruments, Hamden, CT). Each chamber is a closed environment in which no oxygen is allowed in or out. All measurements began at room air $\left(\mathrm{O}_{2}=21 \%=0.341 \mu \mathrm{mol} \mathrm{ml}{ }^{-1}\right)$.

To measure cell consumption, 12-mm-diameter circular coverslips containing adherent primary bone marrowderived nucleated cells (cultured as described above) were placed in four respirometry wells as shown in Fig. 3a. The remaining two wells contained sterile $12-\mathrm{mm}$ coverslips without cells. All wells were filled with $\alpha$-MEM (Sigma Aldrich, St. Louis, MO) supplemented with $20 \mathrm{mM}$ HEPES (Sigma Aldrich, St. Louis, MO). Wells were sealed, and oxygen concentration was measured until all cell-containing wells reached $<1 \%$ oxygen. At the conclusion of the experiment, coverslips were stained for nuclei using $4^{\prime \leftarrow}, 6$-diamidino-2-phenylindole (DAPI) (Vector Labs, Burlingame, CA). Coverslips were then imaged with a fluorescent microscope as seen in Fig. 3b, and a cell count was obtained for each coverslip as previously described by Powell et al. [19] using the Colonyze ${ }^{\mathrm{TM}}$ analysis software. Oxygen consumption rate was calculated as a function of oxygen concentration by taking the derivative of the oxygen concentration as a function of time. The oxygen consumption rate was normalized on a per-cell basis using the cell count from each coverslip.

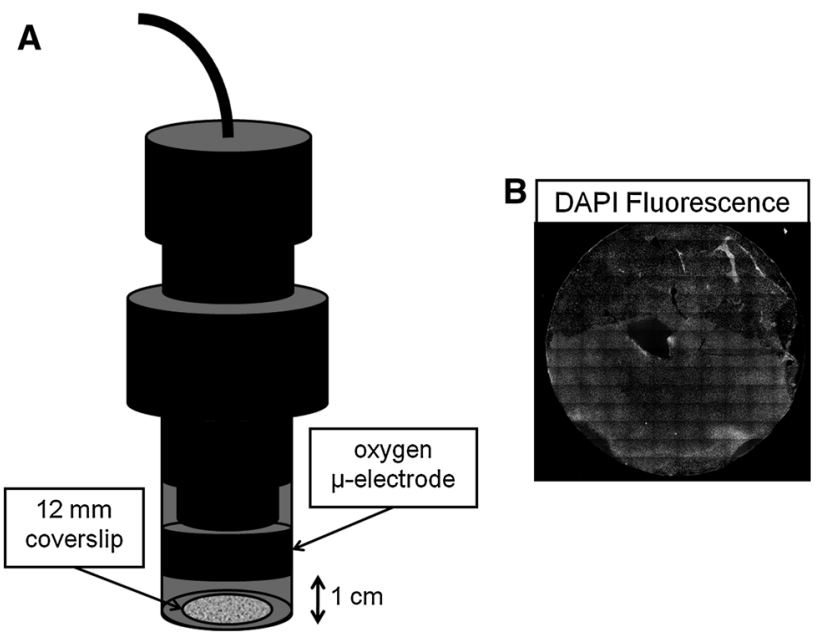

Fig. 3 Respirometry system: a Respirometry well with oxygen microelectrode above a confluent cell layer on a coverslip. b Fluorescent image of nuclei stained with DAPI

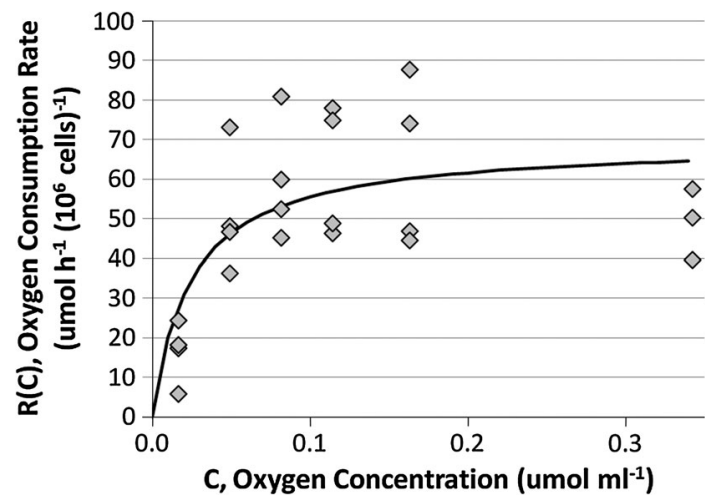

Fig. 4 Oxygen consumption rate of bone marrow-derived nucleated cells: best-fit curve (black line) of saturation kinetics model to data (diamonds) $(N=4) . R^{2}=0.81$

The oxygen consumption rate $R\left(\mu \mathrm{mol} \mathrm{cell}^{-1} \mathrm{~h}^{-1}\right.$ ) of nucleated cells derived from bone marrow is dependent on the oxygen concentration $C\left(\mu \mathrm{mol} \mathrm{ml} l^{-1}\right)$ in the surrounding environment (Fig. 4). The rate of oxygen consumption increased as a function of oxygen concentration up to $0.162 \mu \mathrm{mol} \mathrm{ml}^{-1}$ and leveled off as oxygen continued to increase. To represent the saturation kinetics of these data, we applied a Michaelis-Menten-like model to describe how the oxygen consumption rate varies according to our experimental data $\left(R^{2}=.704\right)$ as:

$R(C)=\frac{\alpha C}{\beta+C}=\frac{69.2 C}{2.46 \times 10^{-2}+C}$

The parameters fit to this model along with the $95 \%$ confidence interval for each parameter are as follows:

$\alpha=69.2[53.3,85.1] \leftarrow$

$\beta=2.46 \times 10^{-2}\left[1.125 \times 10^{-4}, 4.90 \times 10^{-2}\right] \leftarrow$ 


\section{Results}

\subsection{Experimental results}

The OGB was shown to generate oxygen over a period of 48-60 h. Figure 2 demonstrates that over $90 \%$ of the total oxygen generated was released in the first $48 \mathrm{~h}$. Oxygen accumulation was observed in chambers containing OGBs as well as PLGA cylinders during the first $48 \mathrm{~h}$. After $48 \mathrm{~h}$, OGBs continued to accumulate oxygen, while the PLGA cylinders ceased oxygen accumulation. This phenomenon is likely due to the release of air trapped in the materials during gas foaming (cylinders in both cases initially floated to the top of the respirometry chamber). Despite any accumulation seen in PLGA chambers, OGB chambers generally had significantly higher rates of accumulation. These data indicate that oxygen generation is occurring in addition to the release of trapped air during the first $48 \mathrm{~h}$ of the experiment. The variability in magnitude of oxygen generation between each OGB is likely due to the variability in the volume and distribution of trapped air. In some instances, decreases in oxygen concentration (i.e., "negative" oxygen generation or oxygen consumption) were observed. This is likely not oxygen consumption, but rather the result of temperature fluctuations in the water bath, since the control wells (PLGA) always exhibited larger decreases in oxygen than the OGB wells over the same time intervals. To computationally define the oxygen generation by OGBs, the average oxygen release from PLGA cylinders was subtracted from that of OGBs (Eq. 6). In vivo, the combination of air trapped in the PLGA phase, as well as chemical production of oxygen by sodium percarbonate encapsulated in OGBs (represented by $G_{1}$ ), would lead to even further oxygen delivery than that reported in $G(t)$ (Eq. 7). The dynamic range of the electrodes was limiting due to the rapid accumulation of oxygen at early time points. Though not ideal, opening of the chambers to room air at defined intervals allowed for continued quantification of oxygen accumulation by avoiding electrode limitations.

Oxygen consumption of CTPs and their progeny depends on oxygen concentration. Oxygen consumption of bone marrow-derived nucleated cells varies significantly as a function of oxygen tension. The data in Fig. 4 demonstrate that the oxygen consumption rate tends to increase with oxygen tension in bone marrow-derived nucleated cells commonly used for therapy. Similar dependency of oxygen consumption rate on oxygen tension has been observed in other cell populations [7, 8, 25]. Initially, these cells consume oxygen at very low levels given the low oxygen concentrations in the avascular defect. As oxygen is released from the OGBs and as oxygen diffuses in from surrounding tissues, oxygen levels rise, and Fig. 4 shows that cells consume increasingly more oxygen until consumption rates level off at approximately $7-10 \%$ $\left(0.11-0.16 \mu \mathrm{mol} \mathrm{ml}^{-1}\right)$ of oxygen. These data suggest that cells have the ability to regulate their consumption in order to acclimatize to their surrounding oxygen environment. Consequently, it is essential to include in the model the dependence of cellular oxygen consumption rate on oxygen concentration.

\subsection{Simulation results}

In addition to the empirical functions $G(t)$ and $R(C)$, Eqs. 7 and 8 , respectively, the values of all model parameters used for model simulation are specified in Table 1 . Since the parameters $N$ and $\alpha$ always appear as a product in this model, the effect of these parameters on the model output cannot be distinguished. The value for $D$ at $37 \mathrm{C}$ was calculated using interpolation of empirical values for oxygen in water [4]. Values of the geometric parameters were taken from experimental studies [15, 22]. The model involving a partial differential equation was solved numerically using the MATLAB algorithm, "pdepe", to obtain the dynamic changes in the $\mathrm{O}_{2}$ concentration distribution between an OGB placed in the center of the defect and the bone at the periphery of the defect. Simulations were performed to demonstrate the spatiotemporal output of the model, as well as the sensitivity of the model to variable parameters.

OGBs almost always exhibit a nonlinear release profile with some degree of burst release followed by a more stable release rate over a defined time period. Under conditions specified in Table 2, panels A, B, and C in Fig. 5 demonstrate the change in radial distribution of oxygen throughout the defect over $96 \mathrm{~h}$ corresponding to the release rate of oxygen from OGB in the center of the defect

Table 1 Model parameters

\begin{tabular}{|c|c|c|}
\hline Parameters & Description & Quantity \\
\hline$r_{\mathrm{OGB}}(\mathrm{cm})$ & $\begin{array}{l}\text { Radius of oxygen-generating } \\
\text { biomaterial }\end{array}$ & 0.15 \\
\hline$r_{\text {bone }}(\mathrm{cm})$ & Radius of avascular bone defect & $0.5[22]$ \\
\hline$N\left(\right.$ cells $\left.\mathrm{ml}^{-1}\right)$ & $\begin{array}{l}\text { Number of cells per unit volume } \\
\text { in the defect }\end{array}$ & $9.31 \times 10^{5}$ \\
\hline$D\left(\mathrm{~cm}^{2} \mathrm{~h}^{-1}\right)$ & $\begin{array}{l}\text { Diffusion coefficient of oxygen } \\
\text { in water at } 37 \mathrm{C}\end{array}$ & $0.136[4]$ \\
\hline$C_{\text {air }}\left(\mu \mathrm{mol} \mathrm{ml}^{-1}\right)$ & Concentration of oxygen in air & $3.41 \times 10^{-1}$ \\
\hline$C_{\text {bone }}\left(\mu \mathrm{mol} \mathrm{ml} l^{-1}\right)$ & $\begin{array}{l}\text { Concentration of oxygen in } \\
\text { vascularized bone tissue }\end{array}$ & $\begin{array}{l}5.82 \times 10^{-2} \\
{[11]}\end{array}$ \\
\hline$V_{\text {water }}(\mathrm{ml})$ & $\begin{array}{l}\text { Volume of water in the } \\
\text { respirometry well }\end{array}$ & 2 \\
\hline
\end{tabular}


Table 2 Simulation parameter values

\begin{tabular}{llllll}
\hline Simulation \# & $N\left(10^{6}\right.$ cells ml $\left.^{-1}\right)$ & $\alpha$ (unitless) & $r_{\mathrm{OGB}}(\mathrm{mm})$ & $V_{\text {water }} / V_{\mathrm{k}}$ (unitless) & Output \\
\hline 1 & 0.1 & 1 & 15 & 1 & Fig. 5 \\
2 & 0.5 & 1 & 15 & 1 & Fig. 6a \\
3 & 0.1 & 1 & 15 & 1 & Fig. 6a \\
4 & 0.25 & 1 & 15 & 1 & Fig. 6a \\
5 & 0.1 & 5 & 15 & 1 & Fig. 6b \\
6 & 0.1 & 1 & 15 & 1 & Fig. 6b \\
7 & 0.1 & 0.25 & 15 & 1 & Fig. 6b \\
8 & 0.1 & 1 & 7.5 & 1 & Fig. 6c \\
9 & 0.1 & 1 & 15 & 1 & Fig. 6c \\
10 & 0.1 & 1 & 25 & 1 & Fig. 6c \\
11 & 0.1 & 1 & 15 & 5 & Fig. 6d \\
12 & 0.1 & 1 & 15 & 1 & Fig. 6d \\
13 & 0.1 & 1 & 15 & 0.25 & Fig. 6d \\
\hline
\end{tabular}
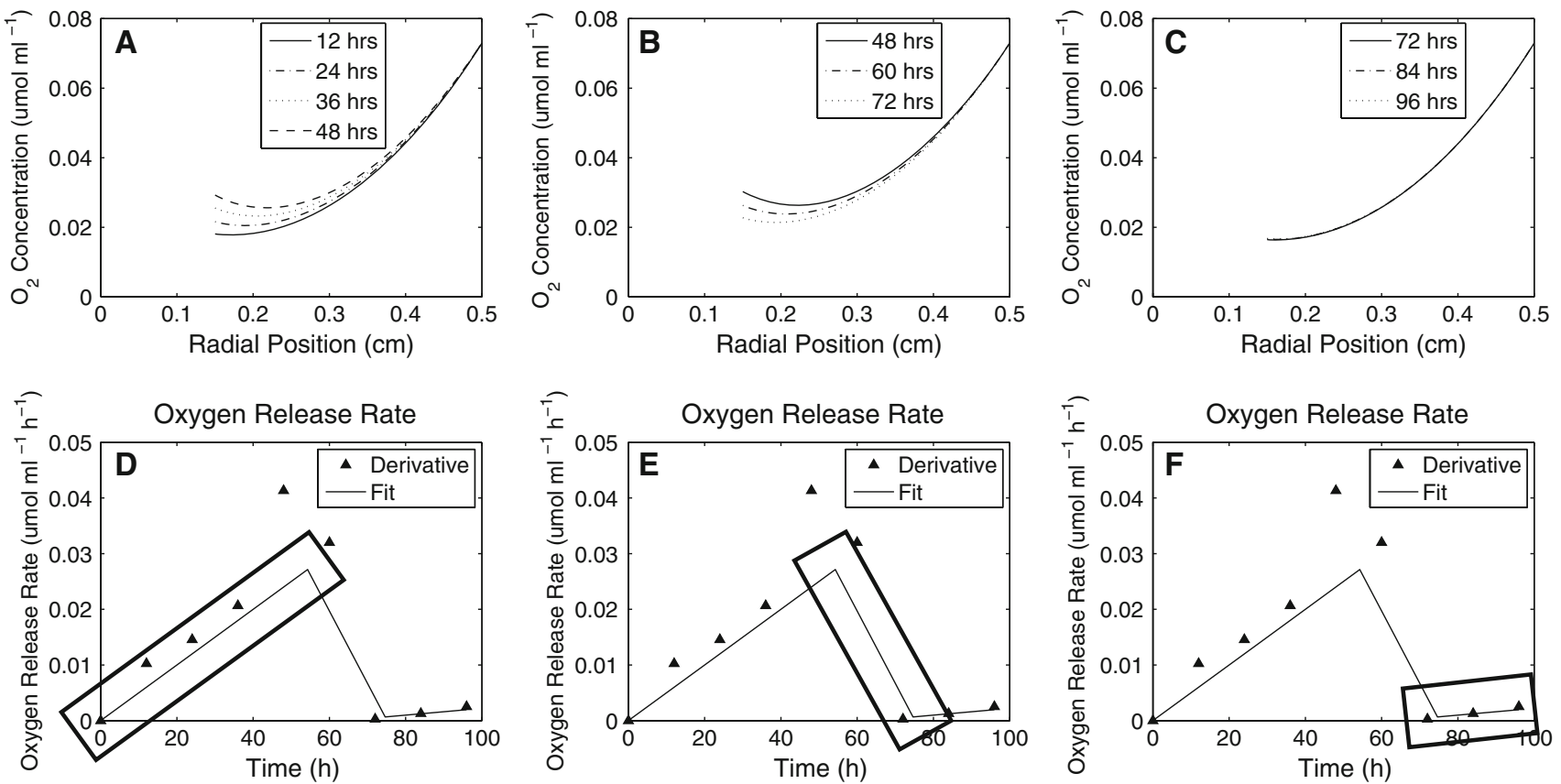

Fig. 5 Model output as a spatiotemporal change in oxygen concentration: Under conditions specified in Table 2, this simulation demonstrates the effect of a time-dependent oxygen generation function $(\mathbf{d}, \mathbf{e}$, and $\mathbf{f})$ on the radial distribution of oxygen in the defect over time $(\mathbf{a}, \mathbf{b}$, and $\mathbf{c})$

(D, E, and F, respectively). Oxygen levels are observed to increase throughout the defect (A) as the OGB oxygen release rate increases from 0 to $48 \mathrm{~h}$ (D). Conversely, oxygen levels are observed to decrease (B) as the oxygen release rate decreases from 48 to $72 \mathrm{~h}$ (E). Little to no change in radial oxygen distribution is observed as the OGB oxygen release rate remains relatively low and constant (C) from 72 to $96 \mathrm{~h}(\mathrm{~F})$.

The simulation in Fig. 5 demonstrates how a timedependent oxygen generation function affects the radial distribution of oxygen in the defect changes over time. Consequently, the model can be used to predict the spatial distribution of oxygen in the defect over time in conjunction with experiments in which key parameters are controlled. As the rate of generation, $G(t)$, increased, a progressively larger fraction of the defect received sufficient oxygen in areas adjacent to the OGB. Conversely, the distribution of oxygen in the defect falls as the generation rate decreases. The temporal changes in oxygen distribution due to OGB release are modulated by the concentration-dependent oxygen consumption. At low generation rates (e.g., $72 \mathrm{~h}$ ), oxygen consumption remains negligible due to minimal oxygen accumulation so that the generation rate still dominates. High oxygen generation rates (e.g., $48 \mathrm{~h}$ ) cause significant oxygen accumulation leading to increased contribution of the oxygen consumption due to 
higher oxygen concentrations. Under this condition, the negative feedback loop of oxygen concentration-dependent consumption prevents runaway oxygen accumulation.

The simulations in Fig. 6 illustrate the predicted sensitivity of the distribution of oxygen to changes in $N$ (cell concentration, $\left(10^{6}\right.$ cells ml $\left.\left.{ }^{-1}\right)\right)$ and $\alpha$ (consumption rate, unitless), $r_{\mathrm{OGB}}$ (i.e., radius $(\mathrm{mm})$ ), and $V_{\text {water }} / V_{\mathrm{k}}$ (relative oxygen generation rate, unitless). Parameter ranges were selected to reflect reasonable physiological and experimental values that demonstrate significant changes in oxygen distribution within the defect. A parabolic-like distribution is evident in all simulations. Analysis of the sensitivity to cell concentration predicts that oxygen tension midway between the OGB surface and the vascularized surface of the defect would fall to near anoxic levels at $48 \mathrm{~h}$ unless the concentration of cells in the defect was reduced to below 100,000 cells ml $^{-1}$. A fourfold reduction in consumption rate could also influence oxygen tension, but only in the setting of cell concentrations at or near 100,000 cells $\mathrm{ml}^{-1}$. Similarly, increasing oxygen generation fivefold could increase the minimum oxygen concentration significantly, but again, only in this critical range of cell concentrations.

\section{Discussion}

\subsection{Simulations to quantify critical parameters}

Simulations of this model can serve as a valuable tool to explore and understand the implications of changes in cell
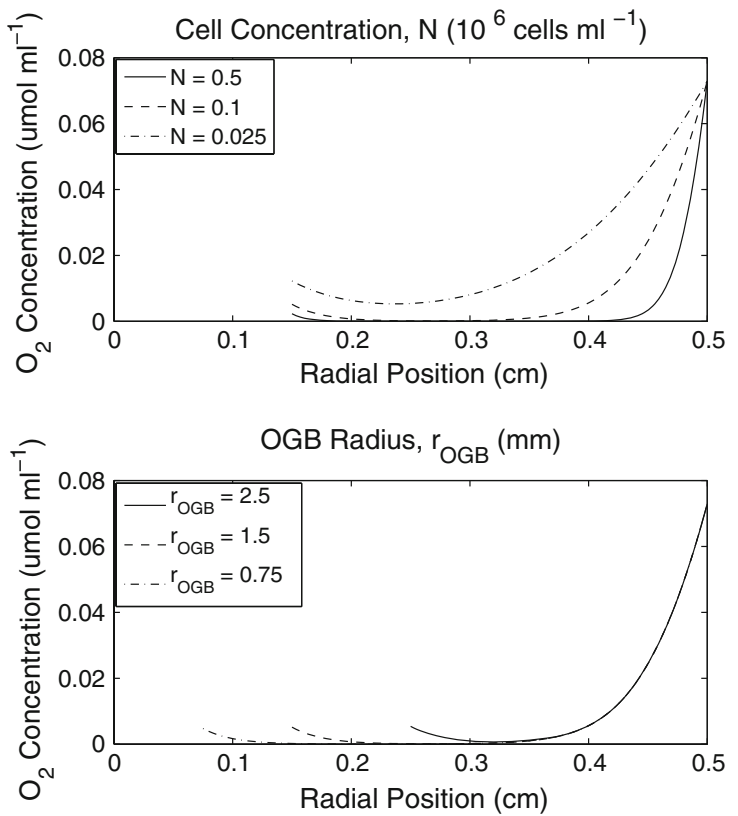

concentration, consumption, and oxygen delivery in a defined bone defect site. These data focus attention on the critical variable of cell concentration as a principle determinant of local oxygen tension within a defect site. Based on measured parameters of oxygen consumption and oxygen generation with our example OGB, these data suggest that therapeutic strategies that involve the delivery of transplanted cells at a concentration of 100,000 cells ml$^{-1}$ or less may be necessary to optimize cell survival. Mathematical models are necessary to make more efficient the task of optimizing the large number of parameters in the design of a tissue engineering construct. A significant time and cost savings can be realized through simulation of an in vivo defect rather than through experimentation only.

\subsection{Model validity}

Assumptions applied in model derivation impact the simulation results. The degree to which each assumption affects the outcomes is important to consider. The assumption that no cell death is occurring has perhaps the most significant effect on simulation results. When oxygen tensions drop to anoxic levels, cells are assumed to be consuming little to no oxygen. In vivo, these cells would likely undergo hypoxia-induced apoptosis. However, with the assumption of no cell death, these cells are capable of consuming oxygen at later simulation time points as oxygen returns to these formerly anoxic regions. Although subpopulations of transplanted cells will be resistant, or even stimulated, at low oxygen tensions, the net effect of this assumption is likely an overestimation of cell oxygen
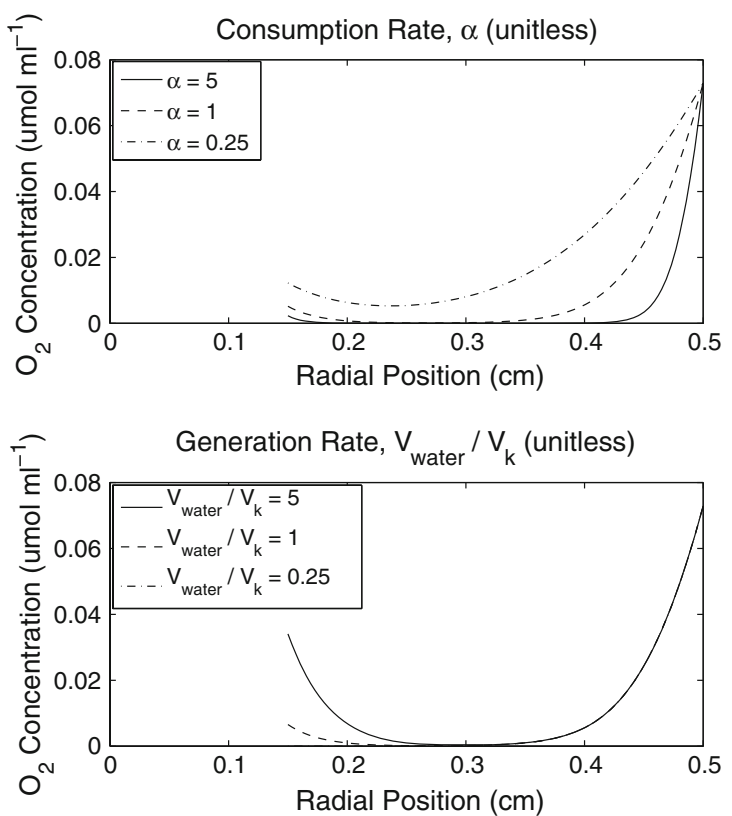

Fig. 6 Effect of model parameters on the radial distribution of oxygen concentration: Plots represent a single time point at $t=48 \mathrm{~h}$. All simulation parameters are defined in Table 2 
consumption and a possible underestimation of how few cells need to be transplanted in order to have a fully oxygenated defect.

The assumption of no proliferation or inflammatory invasion also affects simulation results. In vivo, there is likely some degree of proliferation and inflammatory response. By keeping the number of cells constant in simulations, cellular consumption is likely underestimated and the resultant prediction of oxygen levels in the defect is likely overestimated. As indicated previously, model simulations show the greatest sensitivity to changes in cell concentration. Overall, the assumptions of no cell death or proliferation and no inflammatory response are special cases. If sufficient data were available, these additional processes could also be simulated. However, the overall effect of these cellular changes is likely more neutral since the reduction in cells within the defect due to cell death is offset by the addition of cells due to inflammatory cells or cell proliferation. The balance between the addition and loss of cells will depend largely on the initial cell concentration, which would increase cell loss in the center, and the magnitude of the inflammatory response, which would add cells to the periphery. This combination of events will erode the assumption of a uniform distribution of cells. As a result, the decline in actual oxygen tension at the periphery of the defect may be steeper than predicted, while the decline in oxygen moving away from the OGB may be less. These effects may shift the oxygen distribution to the right.

\subsection{Model flexibility for a wide variety of clinical applications}

This model incorporates defect and scaffold sizes, cellular oxygen consumption rates, cell concentrations, neighboring tissue oxygen concentration, and OGB production rates. Since it accommodates so many construct design parameters, it can simulate a variety of clinically relevant situations. We chose the minimum number of parameters that can be measured independently prior to implantation. In order to simulate a specific set of in vivo phenomena, these parameters characterize the defect and the construct placed in that defect. Simulations can minimize the time and effort to design and fabricate a customized therapeutic populated with the appropriate amount of cells and oxygen-generating materials for a given defect. Although this model does not take into consideration cell proliferation and apoptosis as variables, it is expected that their contributions are relatively small.

Based on the principles of mass transport and reaction processes, this model can easily be adapted and applied to other tissue types. For application to bone defects, the model incorporates diffusion, generation, consumption, and oxygen from surrounding tissues in a closed system. For vascularized tissues, the model would include convection, and for an epithelial sheet of cells, oxygen consumption may not be homogenous.

\subsection{Acceleration of OGB development}

Simulations with this model may accelerate development of OGBs with novel biomaterials. The data in Fig. 2 demonstrate the efficacy of OGBs in delivering oxygen sufficient for approximately $10^{5}$ cells for up to 4 days. Previous work demonstrates the possibility of producing oxygen in significant concentrations on the order of weeks to months using similar materials $[1,5,14,15]$. Once a target defect and therapeutic cell population has been selected, this model can be used to identify the benchmarks (delivery magnitude, delivery rate, cell distribution, geometry, etc.) necessary to create an ideal biomaterial for the defect. This will speed development of new materials from bench to bedside through minimization of in vitro experiments, decreased time and cost of animal studies, and an overall streamlined path to clinical trials.

\subsection{Future studies}

Model simulations can help define the type of critical experiments necessary for designing therapeutic OGBs and cell-loaded constructs. The transplanted cell population must be characterized empirically in order to determine the rate at which cells consume oxygen and how this rate depends on the oxygen concentration in the surrounding environment. Then, model simulations can identify a proper combination of OGB size and oxygen generation rate to address a given defect and transplanted cell population. The results will serve as a benchmark for OGB design.

\subsection{Conclusions}

The mathematical model developed in this study can predict the oxygen distribution for tissue regeneration in a bone defect. This model allows systematic exploration of the likely sensitivity of local oxygen tension to changes in the concentration of cells transplanted, the defect size and geometry, the rate of oxygen delivery in the defect, and the mean oxygen consumption per cell. Simulations highlight the critical parameter of cell concentration $N$ in determining the oxygen concentration throughout the defect. Although changing $\alpha$ has the same effect as changing $N$, the value of $\alpha$ is an intrinsic property of the system and cannot be externally modulated as can be done with $N$. Oxygen consumption of bone marrow-derived nucleated cells is dependent on oxygen concentration and decreases 
as oxygen tension falls below $0.05 \mu \mathrm{mol} \mathrm{ml^{-1 }}$. Oxygen release from OGBs may be considered as a means of modulation of local oxygen tension in a defect site, recognizing that oxygen release is a time-dependent function that must be measured for accurate simulation.

Acknowledgments This work was supported by National Institutes of Health T32 AR050959, the Cleveland Clinic Foundation, and the Armed Forces Institute of Regenerative Medicine (AFIRM). AFIRM is managed and funded through the US Army Medical Research and Materiel Command (MRMC). The AFIRM has additional funding from the US Navy, the Office of Naval Research, the US Air Force Office of the Surgeon General, the National Institutes of Health, the Veterans Administration, the Cleveland Clinic, and local public and private matching funds. The AFIRM contribution to this publication was supported by a subcontract from Rutgers University, Department of Chemistry and Chemical Biology/NJ Center for Biomaterials, under Cooperative Agreement No. WSIXWH-08-2-0034 from the US Department of Defense, US Army Medical Research Acquisition.

Conflict of interest No benefits in any form have been or will be received from a commercial party related directly or indirectly to the subject of this manuscript.

\section{References}

1. Abdi SI, Ng SM, Lim JO (2011) An enzyme-modulated oxygenproducing micro-system for regenerative therapeutics. Int $\mathrm{J}$ Pharm 409:203-205

2. Calori GM, Mazza E, Colombo M, Ripamonti C (2011) The use of bone-graft substitutes in large bone defects: any specific needs? Injury 42(Suppl 2):S56-S63

3. Fayaz HC, Giannoudis PV, Vrahas MS, Smith RM, Moran C, Pape HC, Krettek C, Jupiter JB (2011) The role of stem cells in fracture healing and nonunion. Int Orthop 35:1587-1597

4. Han P, Bartels DM (1996) Temperature dependence of oxygen diffusion in $\mathrm{H}_{2} \mathrm{O}$ and $\mathrm{D}_{2} \mathrm{O}$. J Phys Chem 100:5597-5602

5. Harris LD, Kim BS, Mooney DJ (1998) Open pore biodegradable matrices formed with gas foaming. J Biomed Mater Res 42:396-402

6. Harrison BS, Eberli D, Lee SJ, Atala A, Yoo JJ (2007) Oxygen producing biomaterials for tissue regeneration. Biomaterials 28:4628-4634

7. Heppenstall RB, Grislis G, Hunt TK (1975) Tissue gas tensions and oxygen consumption in healing bone defects. Clin Orthop Relat Res 106:357-365

8. Huang CY, Yuan TY, Jackson AR, Hazbun L, Fraker C, Gu WY (2007) Effects of low glucose concentrations on oxygen consumption rates of intervertebral disc cells. Spine 32:2063-2069

9. Jain HV, Moldovan NI, Byrne HM (2012) Modeling stem/progenitor cell-induced neovascularization and oxygenation around solid implants. Tissue Eng Part C Methods 18:487-495

10. Jonitz A, Lochner K, Lindner T, Hansmann D, Marrot A, Bader R (2011) Oxygen consumption, acidification and migration capacity of human primary osteoblasts within a three-dimensional tantalum scaffold. J Mater Sci Mater Med 22:2089-2095

11. Kiaer T, Dahl B, Lausten G (1992) Partial pressures of oxygen and carbon dioxide in bone and their correlation with bone-blood flow: effect of decreased arterial supply and venous congestion on intraosseous oxygen and carbon dioxide in an animal model. J Orthop Res 10:807-812
12. Malda J, Rouwkema J, Martens DE, Le Comte EP, Kooy FK, Tramper J, van Blitterswijk CA, Riesle J (2004) Oxygen gradients in tissue-engineered PEGT/PBT cartilaginous constructs: measurement and modeling. Biotechnol Bioeng 86:9-18

13. Muschler GF, Midura RJ (2002) Connective tissue progenitors: practical concepts for clinical applications. Clin Orthop Relat Res 395:66-80

14. Muschler GF, Nakamoto C, Griffith LG (2004) Engineering principles of clinical cell-based tissue engineering. J Bone Joint Surg Am 86-A:1541-1558

15. Muschler GF, Raut VP, Patterson TE, Wenke JC, Hollinger JO (2010) The design and use of animal models for translational research in bone tissue engineering and regenerative medicine. Tissue Eng Part B Rev 16:123-145

16. Nandi SK, Roy S, Mukherjee P, Kundu B, De DK, Basu D (2010) Orthopaedic applications of bone graft and graft substitutes: a review. Indian J Med Res 132:15-30

17. Oh SH, Ward CL, Atala A, Yoo JJ, Harrison BS (2009) Oxygen generating scaffolds for enhancing engineered tissue survival. Biomaterials 30:757-762

18. Pedraza E, Coronel MM, Fraker CA, Ricordi C, Stabler CL (2012) Preventing hypoxia-induced cell death in beta cells and islets via hydrolytically activated, oxygen-generating biomaterials. Proc Natl Acad Sci USA 109:4245-4250

19. Powell KA, Nakamoto C, Villarruel S, Boehm C, Muschler G (2007) Quantitative image analysis of connective tissue progenitors. Anal Quant Cytol Histol 29:112-121

20. Sato K, Moy OJ, Peimer CA, Nakamura T, Howard C, Ko SH, Lee TC, Nishiwaki Y (2011) An experimental study on costal osteochondral graft. Osteoarthr Cartil 20:172-183

21. Sheridan MH, Shea LD, Peters MC, Mooney DJ (2000) Bioabsorbable polymer scaffolds for tissue engineering capable of sustained growth factor delivery. J Control Release 64:91-102

22. Takigami H, Kumagai K, Latson L, Togawa D, Bauer T, Powell K, Butler RS, Muschler GF (2007) Bone formation following OP1 implantation is improved by addition of autogenous bone marrow cells in a canine femur defect model. J Orthop Res 25:1333-1342

23. Villarruel SM, Boehm CA, Pennington M, Bryan JA, Powell KA, Muschler GF (2008) The effect of oxygen tension on the in vitro assay of human osteoblastic connective tissue progenitor cells. J Orthop Res 26:1390-1397

24. Wang J, Zhu Y, Bawa HK, Ng G, Wu Y, Libera M, van der Mei $\mathrm{HC}$, Busscher HJ, Yu X (2010) Oxygen-generating nanofiber cell scaffolds with antimicrobial properties. ACS Appl Mater Interfaces 3:67-73

25. Wang L, Wilshaw SP, Korossis S, Fisher J, Jin Z, Ingham E (2009) Factors influencing the oxygen consumption rate of aortic valve interstitial cells: application to tissue engineering. Tissue Eng Part C Methods 15:355-363

26. Wehrhan F, Amann K, Molenberg A, Lutz R, Neukam FW, Schlegel KA (2011) PEG matrix enables cell-mediated local BMP-2 gene delivery and increased bone formation in a porcine critical size defect model of craniofacial bone regeneration. Clin Oral Implants Res 23:805-813

27. Wilson SM, Goldwasser MS, Clark SG, Monaco E, Bionaz M, Hurley WL, Rodriguez-Zas S, Feng L, Dymon Z, Wheeler MB (2012) Adipose-derived mesenchymal stem cells enhance healing of mandibular defects in the ramus of swine. J Oral Maxillofac Surg 70:e193-e203

28. Zhao F, Pathi P, Grayson W, Xing Q, Locke BR, Ma T (2005) Effects of oxygen transport on 3-d human mesenchymal stem cell metabolic activity in perfusion and static cultures: experiments and mathematical model. Biotechnol Prog 21:1269-1280 